\author{
J. Tysarczyk ${ }^{1}$, M. Budzik ${ }^{1,2}$, J. Jumel ${ }^{2}$, K. Imielińska ${ }^{1}$, M. E. R. Shanahan ${ }^{2}$ \\ ${ }^{1}$ Gdańsk University of Technology, Faculty of Mechanical Engineering, Dept. of \\ Material Science and Engineering, Narutowicza 11/12, 80-233 GDANSK, POLAND \\ ${ }^{2}$ Université Bordeaux 1, Laboratoire de Mécanique Physique (LMP)-UMR CNRS \\ 5469, 351 Cours de la Libération, 33405 TALENCE Cedex, FRANCE
}

\title{
RELIABLE METHOD OF ASSESSING FRACTURE PROPERTIES OF ASYMMETRIC BONDED JOINTS
}

\begin{abstract}
Two methods of assessing fracture properties of adhesive joints were studied. Two wedge tests: with continuous deflection and with force measurements were compared. Asymmetric geometry of the bonded joint was considered, i.e. two different plates of aluminium alloys: $\mathrm{Al}-\mathrm{Cu}$ and $\mathrm{Al}-\mathrm{Mg}$, were bonded with epoxy DGEBA adhesive. The analytical model is shown to estimate the values of fracture properties: crack position and critical fracture energy. It was found that both methods allow easy and reliable estimation and comparison of fracture properties, although some differences were observed.
\end{abstract}

Keywords: bonded joints, adhesion, fracture, wedge test.

\section{INTRODUCTION}

Adhesive bonding is one of the oldest yet one of the most innovative techniques of joining materials. The applications of adhesive joints involve many strategic industry sectors such as: transport -with light, energy conserving structures made of modern composite materials, ceramics and metals alloys, [1], civil engineering - with reinforcement of the old structures and building new objects [2], electronics - where adhesive bonding provides the most efficient way of joining small elements [3] etc. This increasing field of applications is also becoming more and more demanding. Progress is made in a few simultaneous pathways such as enhancing the adhesion by the change of chemical and physical structures of bonded surfaces $[4,5]$, change of the adhesive formulation $[6,7]$, development of new models leading to better understanding of the phenomena inside adhesive joints [8] etc. Advancement is also made in the testing methods of adhesion efficiency. Although simple tension and shear test are still among the most common they are not giving valuable results [9], and their use, even for comparison must be carefully considered. Fracture mechanics based tests prove their advantages when more detailed data are required. In addition, fracture mechanics tests allow long term testing, giving opportunity to test the glue (adhesive) or the adhesive joint in hostile environments [10]. 
The aim of this contribution made in cooperation with the Laboratory of Physic Mechanics of the University Bordeaux was to propose and validate two methods of adhesive efficiency testing based on one measuring sensor. Mode I loading conditions where selected since they are the most critical, and by today state of the art in this field they are likely to provide crack initiation and propagation $[11,12]$. The tests were run using constant displacement principle, in which the test begins by imposing some separation distance between two bonded substrates. Herein, the elastic energy stored in the flexible (bent) plate must exceed the value of the fracture energy of the adhesive joint to provide crack initiation. Subsequently, the crack is propagating at a selfdetermined rate, up to the moment when the fracture energy offsets the elastic energy.

\section{EXPERIMENTAL}

\section{Characterization of materials}

In asymmetric wedge test configuration a thin, flexible, aluminium alloy plate was made of (Dural or Avional, AA 2024-T3, Alcoa, USA) of thickness, $h=1.6 \mathrm{~mm}$. It was bonded using pure DGEBA epoxy resin (Araldite Cristal, Bostik, France) to a rigid, or thick, plate of Hydronalium, AA 5754, Alcoa, USA) of thickness $H=6 \mathrm{~mm}$. Young's modulus of the thinner plate, $E$, was obtained from the 3-point bending test, and was evaluated at $68 \pm 5 \mathrm{GPa}$ (recently proved by the TTM ultrasound method). Poisson's ratio, $v$, obtained by ultra-sound TTM (through transmission method), was 0.32 . The properties of the thick member were taken from the supplier, with Young modulus $E_{H}=70 \mathrm{GPa}$ (direct estimation is not required since the properties are not used in all calculation). Since asymmetric geometry convention was used (Fig.1), the relative flexural rigidity must be checked. This is governed by the ratio of the cubes of thickness multiplied by the appropriate Young's modulus, and giving value of $c a$. 53. The terms rigid and flexible are therefore reasonable. The flexible adherend of length, $l=120 \mathrm{~mm}$ was (initially) bonded to the rigid member along length, $l_{\text {adh }}$ while the rigid substrate was of length, $L=180 \mathrm{~mm}$. This gave an initial effective crack length, $a$, as shown (Fig.1). The entire system width was, $b=25 \mathrm{~mm}$.

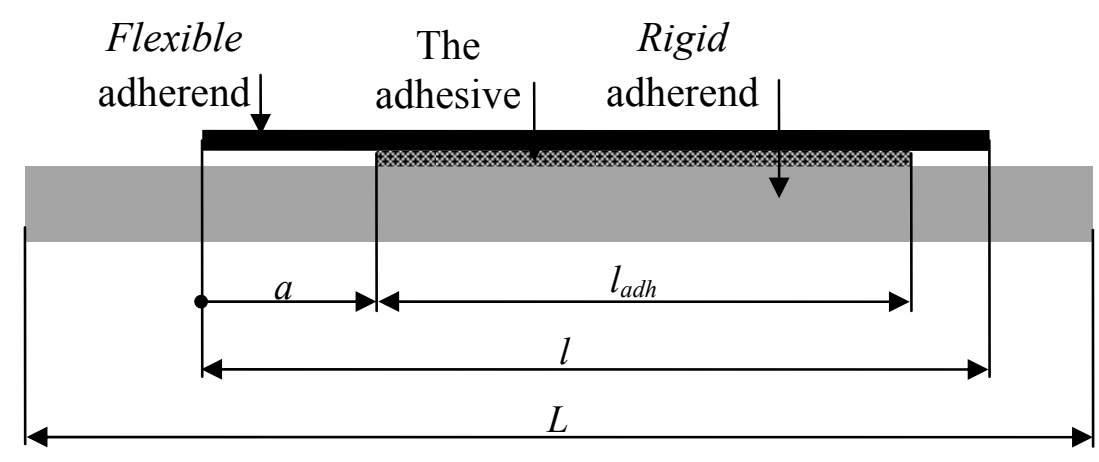

Fig.1. Geometry of the samples

A displacement LVDT sensor (10 $\mathrm{mm}$ range, RDP, UK) or alternatively strain gauge force sensor (500N range, MC Instruments, France) were attached to the central line of 
symmetry, onto the inner surface of the flexible plate (always being in the so-called free zone). The flexible plate was than mounted by the steel chain to the tensile machine. In some of the experiments (detailed below) surface preparation was of no importance, since no adhesive bonding was performed: a controllable, artificial bonded joint was employed instead. This technique is detailed in [13]. In the case of the real joint (ortho)phosphoric acid anodisation (PAA) was performed. After abrading with 2400 grade emery paper, further detergent cleaning, hot air drying and acetone rinsing preceded immersion in an electrochemical bath. Phosphoric acid anodisation (PAA), acknowledged as very effective [e.g. 4, 5], was adopted, using a solution of $10 \%$ (by weight) of phosphoric acid $\left(\mathrm{H}_{3} \mathrm{PO}_{4}\right)$ in deionised water, under a $10 \mathrm{~V}$ direct current potential for 20 minutes at room temperature. The aluminium plate served as anode and a titanium grit - cathode. Next, the surfaces were rinsed in distilled water, hot air dried and finally acetone rinsed. Crosslinking was effected at ambient temperature $\left(\mathrm{ca} .20^{\circ} \mathrm{C}\right)$ for 24 hours under 2 bars pressure and at ca. $55 \%$ RH. Bondline thickness, $e=0.3 \mathrm{~mm}$ was maintained, by inserting PTFE spacers at the two point extremities before crosslinking. The constancy was checked by optical microscope.

\section{Constant Displacement Tests}

In principle, the Constant Displacement Test is made when separation distance between two bonded plates is kept constant during the entire test. In the most common version this refers to the situation when the separation is caused by the wedge insertion (between the plates). After wedge insertion, the separation distance is kept constant since the wedge is left in the initial position. The crack propagates at a self-determined rate. This configuration permits only limited number of parameters to be measured. Consider situation in Fig.2.

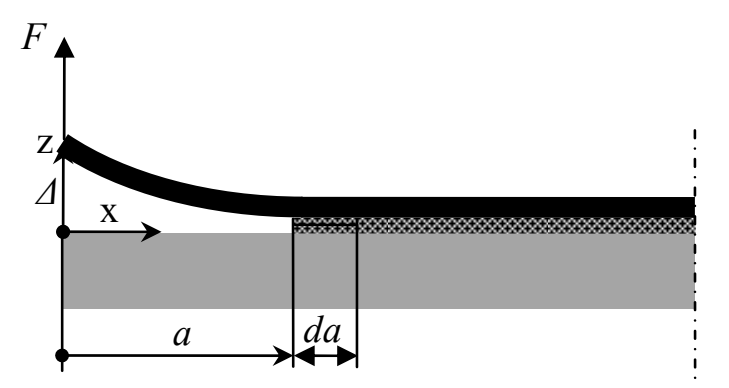

Fig.2. Asymmetric bonded joint fracture test scheme

With the classical Griffith theory [14], and the Irwin-Kies [15] interpretation the fracture energy can be found in form:

$$
G=-\frac{\partial U}{b \partial a}=\frac{F^{2}}{2 b} \frac{d C}{d a}
$$

where: $\quad G$ - energy release rate $\left[\mathrm{J} / \mathrm{m}^{2}\right]$

$C=\Delta F^{-1}-$ compliance

$U$ - elastic energy

$b$ - bonding width 
When values of $\Delta, E$ and $h$ are constant (which is the case in the following study) the energy release rate, or in the case when the crack is propagating, fracture energy $G$ is dependant only on the value of the arm (crack) length $a$. Using Euler-Bernoulli beam theory, the deflection of the bent beam can be given by the equation:

$$
z(x)=\frac{1}{2} \Delta\left[\left(\frac{x}{a}\right)^{3}-3 \frac{x}{a}+3\right]
$$

with the boundary condition:

$$
z(x=0)=\Delta=\frac{1}{3} \frac{F a^{3}}{E I}
$$

where: $I$ - second moment of inertia of the beam cross section, here $I=b h^{3} / 12$.

Thus the crack length can be estimated: directly - from the crack length measurement using, e.g. optical microscope (not accurate, not continuous) [16], by measuring deflection at a certain point [17] (e.g. $z_{i}$ like in Fig.3.) or by measuring change of the force $F$ at any $z(x)$. When the wedge technique is used deflection can be measured easily, the force measurements are not possible, decrease of force must be measured. For that reason we propose different way leading to the constant separation distance allowing both measurements. The initial separation, $\Delta$ is provide by tensile machine so that separation distance can be set using controllable crosshead move. This situation is shown schematically in Fig.3.

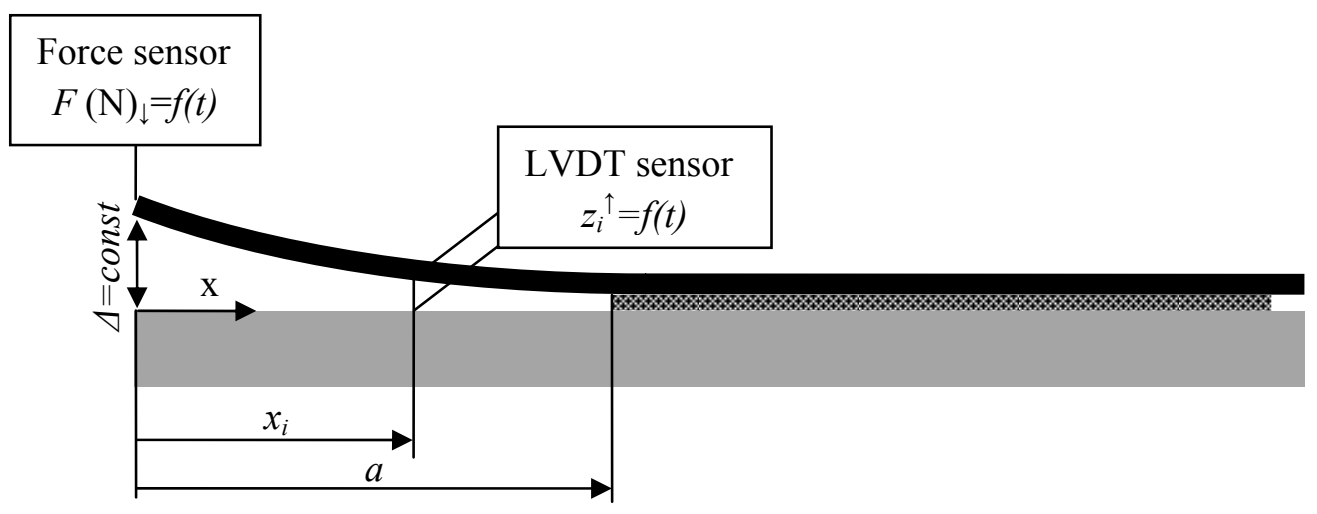

Fig.3. Constant Displacement Tests scheme

As mentioned above, this configuration allows both of the described measurements (LVDT and force) to be held. In addition this can be made simultaneously and both methods could be easily compared.

For the LVDT sensor based at distance $x_{i}$ from the $z$ origin and measuring deflection of the beam $z_{i}$ crack length can be found as: (equation 2 has three roots, two complex and with only one being real): 


$$
a=x_{i}\left\{2 \cos \left[\frac{1}{3} \cos ^{-1}\left(\frac{z_{i}}{\Delta}-1\right)+\frac{4}{3} \pi\right]\right\}^{-1}
$$

Although, theoretically, crack length can be estimated from any sensor based within $\mathrm{x}<0, \mathrm{a}>$, the sensitivity of the method varies depending on distance $x_{i}$. The value of $\mathrm{x}_{\mathrm{i}}$ providing the highest sensitivity will maximize the derivative of $z_{i} / a$ from equation 2 . Thus:

$$
x_{i_{\text {optimum }}}=\frac{a}{\sqrt{3}}
$$

$>$ In the case of crack length estimation using force measurement eq. 3 can be transformed to give:

$$
a=\sqrt[3]{3 \frac{E I \Delta}{F}}
$$

Finally, in both cases the fracture energy equation is in the form:

$$
G=\frac{3}{8} \frac{E \Delta^{2} h^{3}}{a^{4}}
$$

\section{RESULTS AND DISCUSSION}

\section{Artificial Crack Tip Test}

Before real bonded joints testing a sequence of calibrating tests have been carried out. This was made in order to calibrate introduced methods. The real crack lengthestimated crack length calibration curves were obtained and calibration equations are given. This was made using Artificial Crack Tip Test [13] where the crack length was set using screw based, collar like clamping system (so that crack length can be measured directly e.g. using calliper gauge like in that case). The schematic representation of the Artificial Crack Tip Test principle is shown in Fig.4.
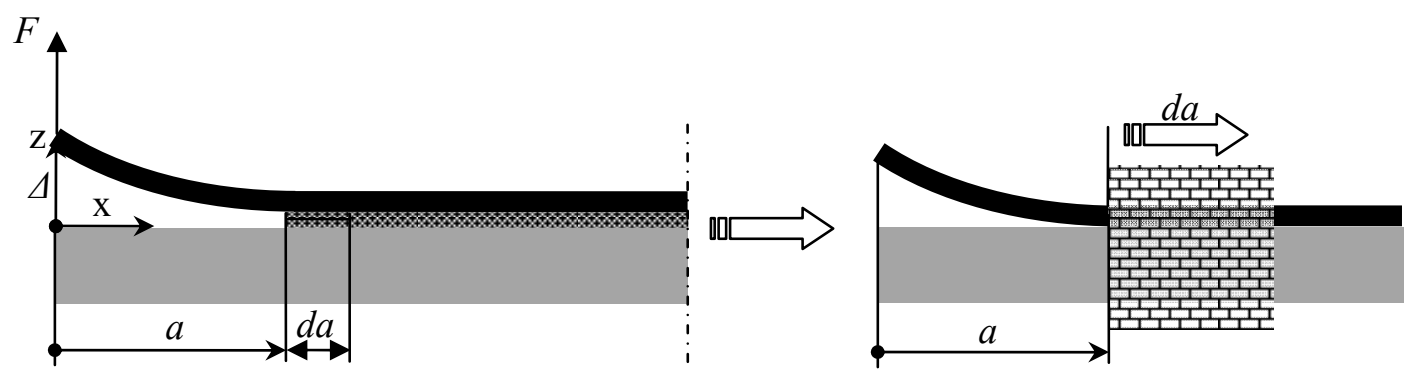

Fig.4. Actual bonded joint (left) and its artificial version 
Such movable platform, represented by brick wall in Fig.4, allows any crack length to be used and thus estimated crack length calibration curve can be easily found. The advantage of the method is that the efficiency of the measurement is checked without using an actual bonded joint.

Assessment of the dissimilarities between crack lengths results obtained from two methods was also important. The same aluminium substrates as in real joints were used. The test was repeated at least three times for different plate, separation distance configuration. The results in the form of the calibration curves are shown in Fig.5. The sensors electric noise was also verified. It was found that the noise is constant within the whole range of the sensors and neglecting it is of no consequence (size of the used data points). The linear relation between the real and estimated crack length were found, giving simple calibration coefficient of 0.97 and 1 for the LVDT and force estimations respectively.

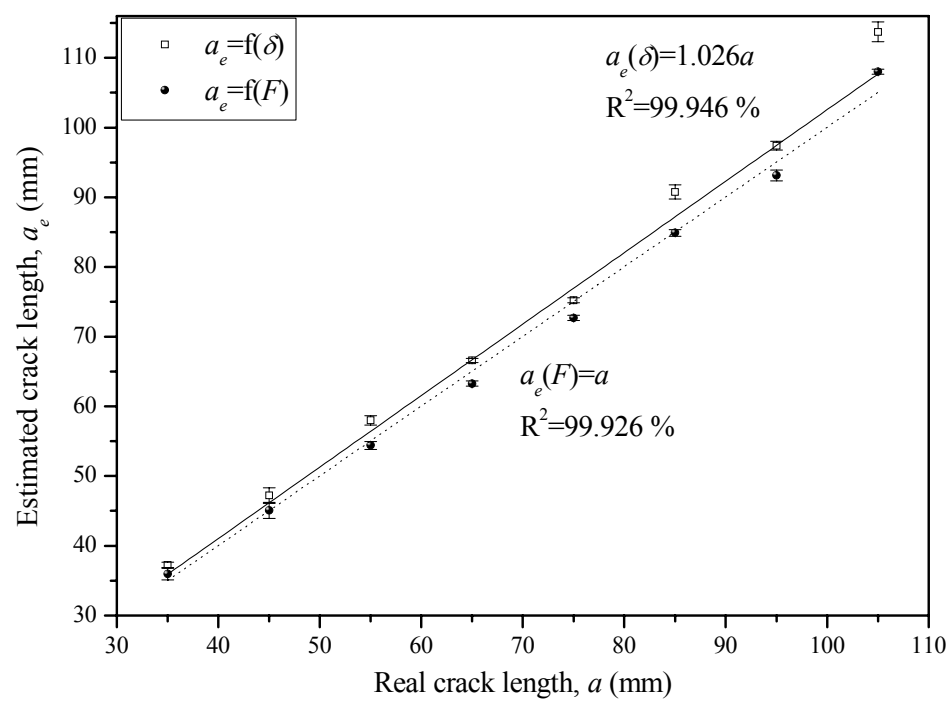

Fig.5. Results from artificial crack tip test

\section{Real Joint Testing}

Since the model and method assumptions were validated, real Al/epoxy/Al joints were tested. The raw results from the force and displacement based measurements are shown in Fig.6.
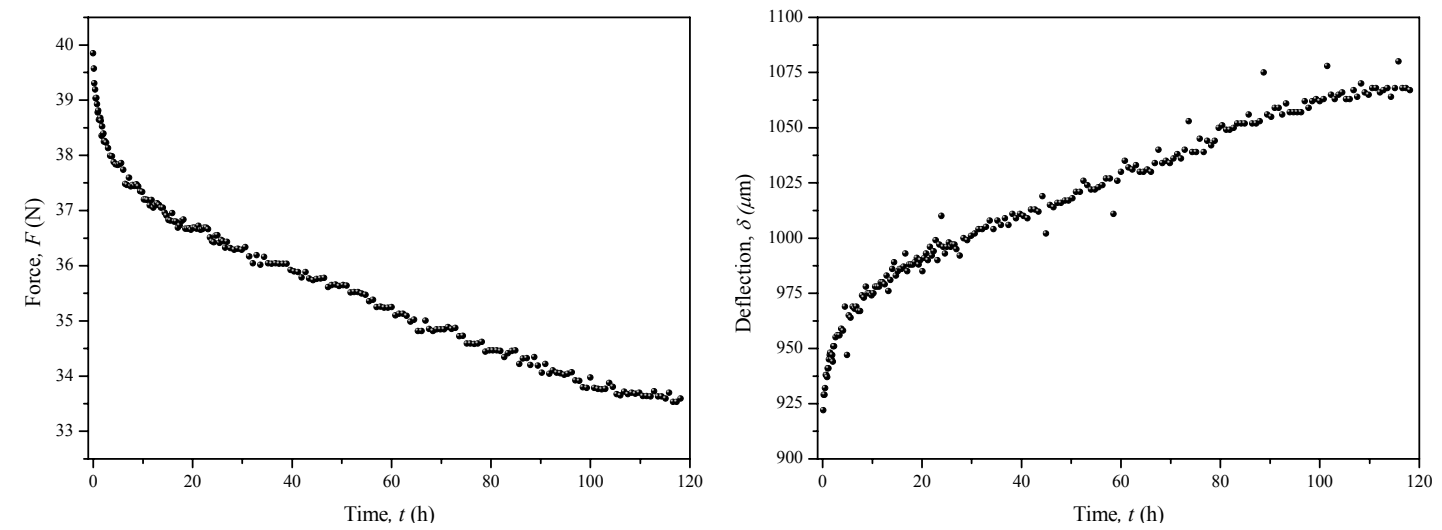

Fig.6. Results from the force (left) and displacement measurements (right) versus time 
Some comments are required about the sensitivity of the measurements. The force sensor used was $500 \mathrm{~N}$ range. Within the test $c a .6 .5 \mathrm{~N}$ change was registered. With $0.1 \mathrm{~N}$ of the force measurement uncertainty possible error of $\pm 1.5 \%$ can be introduced. The LVDT sensor was of $10 \mathrm{~mm}$ range. The change of deflection $z_{i}$ was of $c a .135 \mu \mathrm{m}$ with the $c a .2 \mu \mathrm{m}$ uncertainty of the measurement, leading to approximately the same error as in the previous method. The differences between two methods are shown in Fig.7. Crack length taken directly from the estimation is shown (left), and after using calibration coefficient (right).
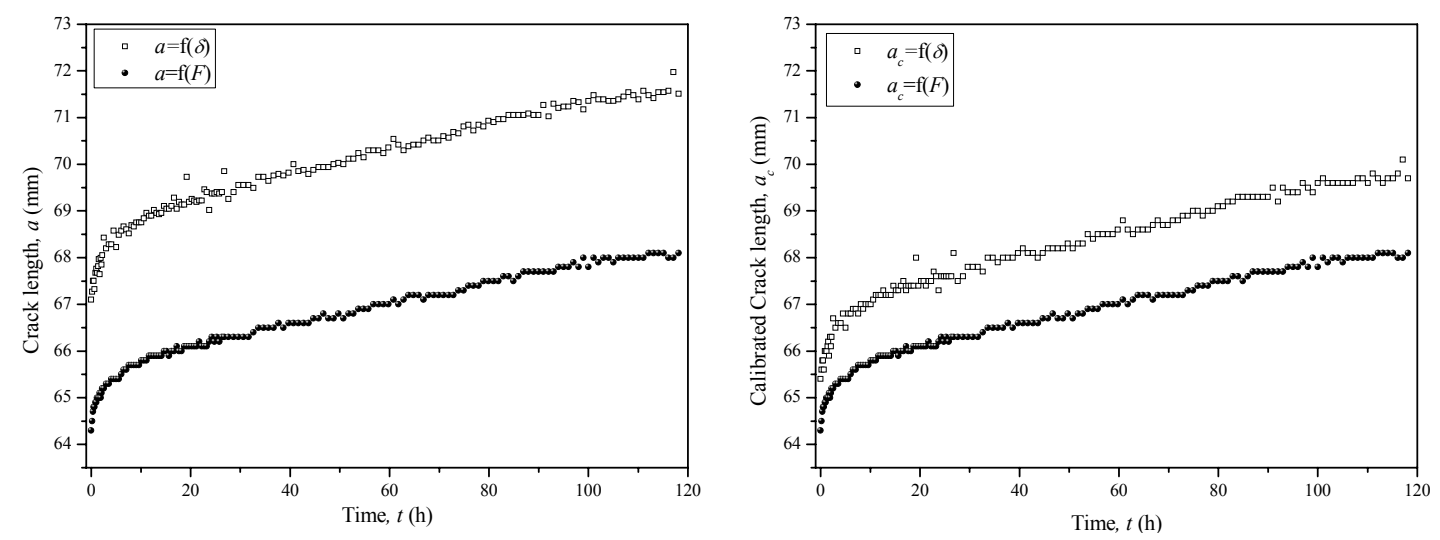

Fig.7. Crack length vs. time from direct estimation using equations (left), and after calibration (right)

Despite calibration, small mismatch of $c a .2 \%$ between two methods exists. This fact cannot neglect any of the methods reported. Simple Euler-Bernoulli method was used. In addition any $2 \mathrm{D}$ effects, like root rotation, or $3 \mathrm{D}$ effects, like crack front curvature are explained. This can indeed lead to this slight difference. Moreover, both sensors are based in different places. Force sensor is attached to the bent adherend extremity, where no anticlastic curvature exists, but root rotation effect can be more pronounced. The LVDT sensor is much closer to the crack tip where anticlastic effect plays important role but the root rotation will be smaller. In fact, we are not able to consider all the effects, the state of the art does not allows that. The important fact is that this initial difference is kept almost constant during the entire crack propagation providing that the crack kinetics can be observed in each method. The crack kinetics is shown in Fig.8 and has very classical form.
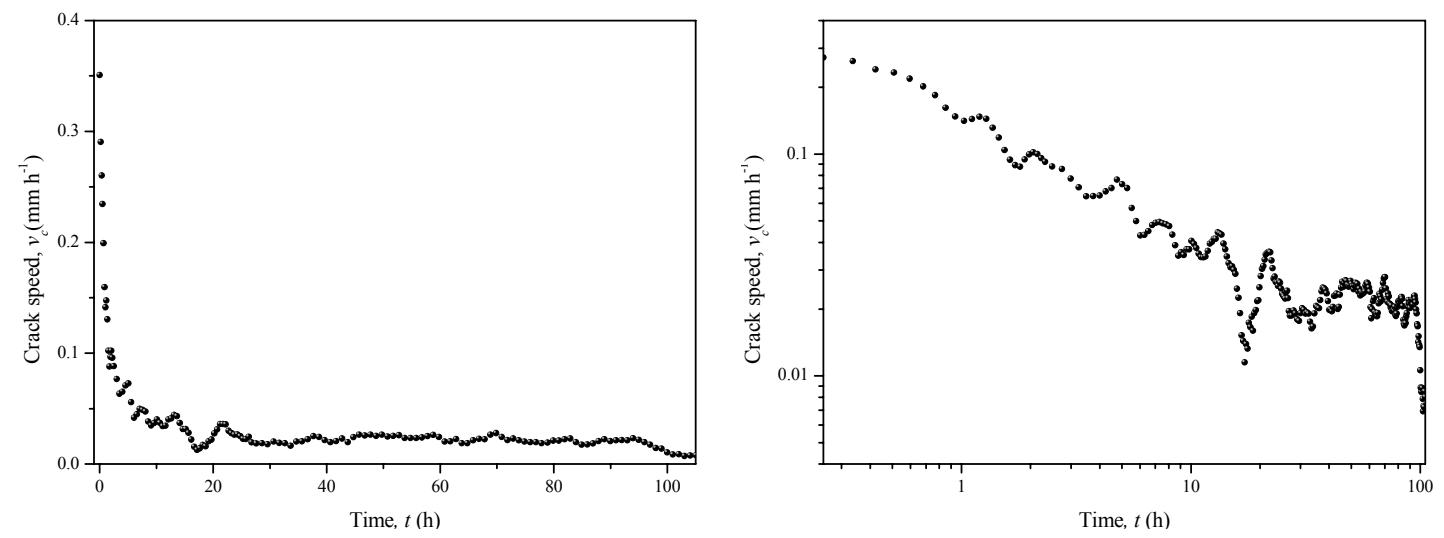

Fig.8. Crack speed change given in linear (left) and log (right) time scales 
Initially, when the energy supplied is highest the crack is propagating at high speed. Than, specifically in log scale, we see continuous slowing down, so normal behaviour of the joint bonded with the epoxy adhesive is observed. However, due to continuous measurements some second order changes can be found, which is not very common in the literature. Finally, the most important and interesting, fracture energy-crack speed characteristics could be drawn. These are shown in Fig.9.
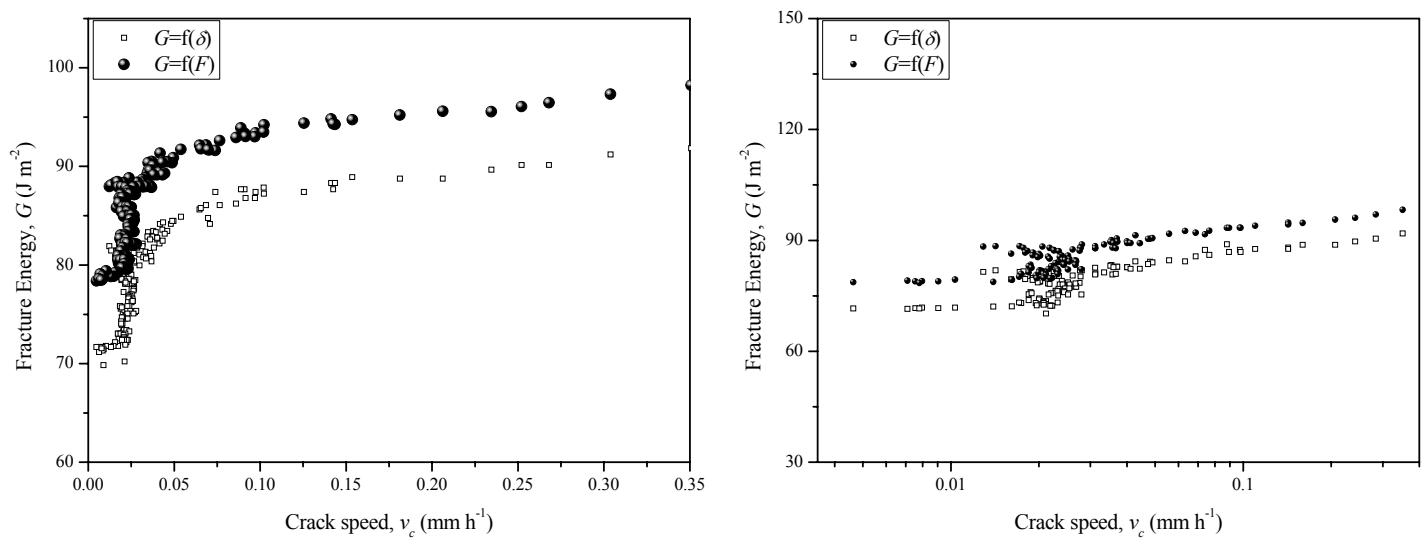

Fig.9. Fracture energy given by the two compared methods in linear (left) and semi-log scales (right)

Continuous measurements are possible using the introduced method. Thus, fracture energy can be given at any crack speed. Results illustrated are made in the linear (left) and semi-log (right) scales. Once more they prove what was previously observed that fracture energy is linearly proportional to the log of the crack speed. What we can also notice is that at crack speed of $c a .0 .02 \mathrm{mmh}^{-1}$ we have highest experimental data density which can be important from statistical point of view, where this speed can be treated as a most probable in situation when the joint is not monitored. The difference of $c a .12 \%$ between the energy values given by the two methods is here emphasized. This comes from the scaling ratio of the power of 4 between the energy and crack length (eq. $6)$.

\section{CONCLUSIONS}

The adhesion efficiency was studied between two aluminium alloys plates bonded with an epoxy DGEBA adhesive. Two one-sensor methods of assessing fracture properties of the adhesive asymmetric joints were proposed and compared. The following conclusions can be drawn:

1. One sensor fracture tests are possible.

2. Fracture energy value was found different depending on the method.

3. Methods proposed, based on Constant Displacement Test principle, could be used to give fast and reliable results which was proved by the Artificial Crack Tip Test.

4. The difference between the methods was found constant and dependent on the tested system. 
5. Further advancement of the $2 \mathrm{D}$ and $3 \mathrm{D}$ effect studies within the adhesive joints are required.

\section{ACKNOWLEDGEMENT}

This research work was supported by the European Union - European Social Fund, the system project of the Pomorskie Voivodeship "InnoDoktorant - Scholarships for PhD students - II edition”.

\section{REFERENCES}

1. Wegman R., Tullos T. R.: Handbook of Adhesive Bonded Structural Repair, Noyes Publications, 1992.

2. Täljsten B.: The Importance of Adhesive Bonding-An histiroci overview and future possibilities, Proceedings of the International Symposium on Bond Behaviour of FRP in Structures, ed. Chen and Teng, International Institute for FRP in Construction, 2005.

3. Licari J. J., Swanson D. W. : Adhesive Technology for electronic applications, William Andrew Publishing, 2005.

4. Rider A. N., Olsson-Jacques C. L., Arnott D. R.: Influence of Adherend Surface Preparation on Bond Durability, Surf. Interface Anal. 27, pp. 1055-1063, 1999.

5. Critchlow G., Brewis D. M.: Review of surface pretreatments for aluminium alloys, J. Adhesion and Adhesives, 16, pp. 255-275, 1996.

6. Irfan M. H.: Chemistry and Technology of Thermosetting Polymers in Construction Applications, Kluwer Academic Publishers, 1998.

7. Jin F. L., Park S. J.: Improvement in fracture behaviors of epoxy resins toughened with sulfonated poly(ether sulfone), Polymer Degradation and Stability, pp. 509-514, 2007.

8. Aymerich F., Lecca G., Priolo P.: Modelling of delamination growth in composite laminates by the virtual internal bond method, Composites: Part A 39 pp. 145-153, 2008.

9. Dillarad D. A.: Adhesion Science and Engineering, A. V. Pocius [ed.], Eslevier Science, pp.1-45, 2002.

10. Bujanda A., Copeland C., Dibelka J., Forster A., Holmes L., Jensen R., Kosik W., McKnight S., Koellhoffer S., Gillespie Jr. J.: Analysis of Adhesively Bonded Ceramics Using an Asymmetric Wedge Test, ARL-TR-4665, 2008.

11. Goland M., Reissner E.: The stresses in cemented joints, J. Appl. Mech., Trans. ASME, 66, A17-A27, 1944. 
12. Carpenter W. C.: Stresses in bonded connections using finite elements, Int. J. Numer. Methods Engng., 15, pp. 1659-1680, 1980.

13. Budzik M., Jumel J., Imielinska K., Shanahan M. E. R.: Accurate and continuous adhesive fracture energy determination using an instrumented wedge test, International Journal of Adhesion and Adhesives 29, pp. 694-701, 2009.

14. Griffith, A. A.: The phenomena of rupture and flow in solids, Philosophical Transactions of the Royal Society of London, A 221, pp. 163-198, 1922.

15. Irwin G.: Analysis of stresses and strains near the end of a crack traversing a plate, Journal of Applied Mechanics 24, pp. 361-364, 1957.

16. Aglan H, Abdo Z.: An innovative approach to fatigue disbond propagationin adhesive joints, J Adhes Sci Technol, 10, pp.183-98, 1998.

17. Sener J.-Y., Ferracin T.; Caussin L., Delannay F.: On the precision of the wedge-opened double cantilever beam method for measuring the debonding toughness of adhesively bonded plates, International Journal of Adhesion and Adhesives, Volume 22, Number 2, pp. 129-137, 2002. 